\title{
ON THE EXISTENCE OF FINITE COLLAPSING SYSTEMS OF PLANE VORTICES
}

\author{
MAREK LEWKOWICZ \\ Wroctaw University of Technology, Department of Numerical Flow Modeling, Wroctaw, Poland \\ e-mail: marek.lewkowicz@pwr.wroc.pl
}

\begin{abstract}
A rigorous criterion for a finite system of plane vortices to be close to the set of all collapsing configurations is given. The criterion is a consequence of a general theorem (based on the topological degree of a map) on the existence of solutions of the nonlinear systems of equations, combined with some analytical bounds for derivatives of the equations satisfied by collapsing configurations. The criterion can be used to prove the existence of collapsing configurations of vortices for many circulations for which the existence theory of O'Neil does not work. A detailed application of the criterion to a sample collapsing system of seven vortices is given.
\end{abstract}

Keywords: collapsing vortex configurations

\section{Introduction}

The evolution of finite systems of plane vortices has been studied since Helmholtz (1858). Of particular interest are systems that collapse, that is converge to a point in finite time during evolution. All three-vortex collapsing systems were described by Groebli (1877). Some special, explicit examples of collapsing systems of four and five vortices were provided by Novikov and Sedov (1987). The existence of collapsing systems of $n$ vortices was proved by O'Neil (1987) for arbitrary $n$. The proof makes use of a system of algebraic equations that self-similar collapsing configurations should satisfy (cf. Lemma 4 and Corollary 1). O'Neil proved that for some circulations, the set of solutions is a non-empty algebraic curve in the configuration space. The proof is non-constructive. In particular, it does not allow one to conclude that a point where the equations are satisfied approximately is in fact close to the solution to the equations.

We proposed in Lewkowicz and Kudela (2012) to seek high-accuracy numerical approximations of collapsing configurations by solving numerically the equation $f(z)=0$ (the map $f$ given by Formula (2.5)) using a two-step relaxation procedure. In the first step we apply the steepest descent method to the function $g(z)=|f(z)|^{2} \in \mathbb{R}$. The method seems to be convergent for many randomly taken initial points. When a certain level of accuracy is attained, the method becomes very inefficient. Fortunately, Newton's method, which could be divergent for a random initial point, is usually convergent then. It rapidly produces a point where O'Neil's equations are satisfied with a very high accuracy. Despite the high accuracy obtained here, strictly speaking, the point we found cannot be called an approximate solution since it is not clear whether any true solution is close to it. A rigorous proof of the existence of a true solution close to the point we have found follows from the criterion which is the main aim of this paper, and whose proof will be presented in Section 4 .

Our criterion is a consequence of a general theorem on the existence of solutions to nonlinear systems of equations (see Section 3. The importance of theorems of this type comes from the fact that finitely many inequalities (which may be verified numerically) imply rigorously the equality $f(x)=0$, which in principle cannot be verified numerically. Our proof of that theorem is based on the notion of the topological degree of a map. Its application to collapsing configurations of 
vortices is made possible by some analytical bounds for the derivatives of the equations satisfied by the collapsing configurations.

The criterion can be used to prove the existence of collapsing configurations of $n$ vortices (for small values of $n$ ) in a way independent of the original proof given by O'Neil (1987). Although we rely on the equations introduced by O'Neil, our existence argument differs from his, as we use topological degree and numerical approximation instead of algebraic geometry. To be more precise, for many circulations we are able to find numerically (by the aforementioned relaxation procedure) explicit configurations with rational decimal coordinates which satisfy the assumptions of the criterion. The conclusion provided by our criterion states then that some explicitly given neighbourhood of the configuration contains another configuration which collapses in the rigorous sense. This proves that collapsing systems exist for these circulations. In Section 6, we perform detailed calculations along these lines for a sample system of seven vortices. As a conclusion we obtain the existence of collapsing systems of seven vortices for the circulation considered there. Clearly, this approach works only for a specific number of vortices chosen for calculations, while O'Neil's approach works for an arbitrary number of vortices. Nevertheless, our approach has the advantage of specifying the accuracy explicitly. Moreover, it works for diverse circulations, not necessarily meeting the conditions required by O'Neil (1987) Theorem 7.1.1. Also, the collapsing configurations we obtain have no direct relation to any collinear rotational configuration, whose existence is required by O'Neil (1987) Theorem 7.4.1.

\section{Basic notions and facts}

We start with a review of some basic notions and facts. For this basic material one can result to e.g. O'Neil (1987), Aref et al. (1992), Garduno and Lacomba (2007).

The notion of a system of discrete vortices is motivated by a discretization procedure in which a smooth vorticity field is divided into regions $U_{i}$, each of them replaced by a point vortex $z_{i}$ given a suitable circulation $\kappa_{i}$. The equations of motion of the system $z(t)=\left(z_{1}, \ldots, z_{n}\right) \in \mathbb{C}^{n}$ of $n$ vortices are

$$
\frac{d z_{l}(t)}{d t}=V_{l}(z(t))=\mathrm{i} \sum_{j=1(\neq l)}^{n} \kappa_{j} \frac{z_{l}-z_{j}}{\left|z_{l}-z_{j}\right|^{2}}
$$

with nonzero real numbers $\kappa_{j}$.

Following O'Neil (1987) let us introduce the size $S(z) \in \mathbb{R}$ and the moment of vorticity $M(z) \in \mathbb{C}$ by

$$
S(z)=\sum_{k} \kappa_{k}\left|z_{k}\right|^{2} \quad M(z)=\sum_{k} \kappa_{k} z_{k}
$$

Two systems of vortices $z$ and $w$ are said to form the same configuration if they are similar, i.e., $w_{k}=a z_{k}+b$ for some complex $a, b$ with $a \neq 0$. The configuration space for $n$ vortices has dimension $2 n-4$ since the group of similarities $z \rightarrow a z+b$ is four-dimensional. It is easy to see that $w_{k}=a z_{k}+b$ implies $V(w)=\frac{1}{\bar{a}} V(z)$ and therefore any trajectory $t \rightarrow z(t)$ gives a trajectory $t \rightarrow a z\left(\frac{t}{|a|^{2}}\right)+b$ with initial conditions $a z(0)+b$. We express this by saying that similar systems are dynamically equivalent. A system $z(0) \in \mathbb{C}^{n}$ is said to collapse if its trajectory $z(t) \in \mathbb{C}^{n}$ represents a one-parameter family of vortex systems convergent to a point at some $t_{\infty}>0$. More precisely, all components $z_{k}(t), 1 \leqslant k \leqslant n$, are required to converge to a common (independent of $k$ ) limit in $\mathbb{C}$ as $t \rightarrow t_{\infty}^{-}$. All systems similar to $z(0)$ also collapse; thus the collapsing families are at least four-dimensional. In fact, as O'Neil's work shows, quite often they are five-dimensional. It is more convenient to speak of collapsing configurations instead of 
collapsing systems: the families described by O'Neil are one-dimensional (form algebraic curves in the configuration space).

A system is called self-similar if it remains similar to the initial state during evolution. This amounts to say that the trajectory $z(t)$ is a fixed point in the configuration space. One also says its configuration is stationary. It is believed that any collapsing system must be self-similar. We consider only self-similar collapsing systems in this paper. It is easy to express analytically the trajectory of any self-similar system (cf. O’Neil, 1987; Lewkowicz and Kudela, 2012).

Lemma 1. Let $w$ be a system of vortices and $z(t)$ the trajectory starting at $w$. The following conditions are equivalent.

- (a) The system $w$ is self-similar.

- (b) $V_{k}(w)-V_{l}(w)=\omega\left(w_{k}-w_{l}\right)$ for some $\omega \in \mathbb{C}$.

- (c) $V_{k}(w)=\omega w_{k}-p$ for some $\omega, p \in \mathbb{C}$.

- (d) The system $w$ belongs to one of the following five classes.

- (i) stationary: $V_{k}(w)=0$, or equivalently $z(t)=w$,

- (ii) translatory: $V_{k}(w)=v \neq 0$ or equivalently $z(t)=w+t v$,

- (iii) rotational: $V_{k}(w)=\mathrm{i} \lambda\left(w_{k}-p\right), \lambda \in \mathbb{R}^{*}, z(t)=p+\mathrm{e}^{\mathrm{i} \lambda t}(w-p)$,

- (iv) collapsing: $V_{k}(w)=\omega\left(w_{k}-p\right), \operatorname{Re}(\omega)<0$,

$$
z(t)=p+\sqrt{2 \operatorname{Re}(\omega) t+1} \mathrm{e}^{\mathrm{i} \frac{\operatorname{Im}(\omega)}{2 \operatorname{Re}(\omega)} \ln (2 \operatorname{Re}(\omega) t+1)}(w-p)
$$

- (v) expanding: $V_{k}(w)=\omega\left(w_{k}-p\right), \operatorname{Re}(\omega)>0, z(t)$ given by the same formula as for the collapsing system.

Minor terminology remarks: the systems that we call rotational are usually referred to as relative equilibria in the literature. Moreover, we distinguish between the collapsing and expanding systems. Usually they are all called collapsing, although a system with $\operatorname{Re}(\omega)>0$ "collapses" in negative time. Nevertheless, its image under any orientation-reversing isometry provides a system collapsing in positive time.

Note that a collapsing trajectory is defined for $t \in\left(-\infty, t_{0}\right), t_{0}=\frac{-1}{2 \operatorname{Re}(\omega)}>0$, and that in fact $\lim _{t \rightarrow t_{0}^{-}}\left|z_{k}(t)-p\right|=0$. Note also that $\omega$ in (b), (c) and (d) is the same. The zero value of $\omega$ corresponds to (d-i,ii). A system is collapsing, expanding or rotational (d-iii,iv,v) iff it satisfies (b) (or equivalently (c)) with $\omega \neq 0$.

Now we specify and reformulate algebraic conditions that self-similar collapsing configurations and their circulations should satisfy. The proofs can be found in O'Neil (1987). We start with conditions for circulations. For a circulation $\kappa=\left(\kappa_{i}\right)_{i=1, \ldots, n} \in \mathbb{R}_{*}^{n}$ define the angular momentum $L$ and the total circulation $\sigma$ by

$$
L=\sum_{i<j} \kappa_{i} \kappa_{j} \quad \sigma=\sum_{i} \kappa_{i}
$$

Lemma 2. If a collapsing configuration exists, then necessarily

$$
L=0 \quad \sigma \neq 0
$$

From now on we assume that condition (2.4) is satisfied.

Lemma 3. If $\sigma \neq 0$, then any system $z$ admits a unique translation image $w=z-p$ (for some $p \in \mathbb{C})$ with $M(w)=0$. 
It follows that each configuration class contains a representative satisfying $M=0$ and that the representative is unique up to a complex factor. In other words, the configuration space is in a natural one-to-one correspondence with the complex projective space $\mathbb{C} P(W)$ over the kernel $W$ of the linear map $M$. This will be of practical importance since now a set of configurations can be specified by a system of homogeneous equations, one of them being $M=0$.

Lemma 4. (See O'Neil (1987), Lemma 1.2.4 and Lemma 1.2.7) Suppose that $L=0, \sigma \neq 0$, $M(w)=0$. Then the following conditions are equivalent

- (a) $w$ is self-similar and expanding, collapsing or rotational.

- (b) $V_{k}(w)-V_{l}(w)=\omega\left(w_{k}-w_{l}\right)$ for any $k, l$ and some non-zero $\omega \in \mathbb{C}$.

- (c) $V_{k}(w)=\omega w_{k}$ for any $k$ and some non-zero $\omega \in \mathbb{C}$.

- (d) at least one $V_{j}(w)$ is non-zero and for some fixed $k$ the equality $w_{l} V_{k}(w)=w_{k} V_{l}(w)$ is satisfied for all indices $l$.

- (e) at least one $V_{j}(w)$ is non-zero, $S(w)=0$ and furthermore for some fixed $k$ the equality $w_{l} V_{k}(w)=w_{k} V_{l}(w)$ is satisfied for at least $n-3$ indices $l$ different from $k$.

Since at most one coordinate of a vortex system can be zero, we get the following important conclusion, which we refer to as the O'Neil equations. Solving these equations is our principal aim.

Corollary 1. (See O'Neil (1987), Lemma 1.2.7) Each configuration in $\Sigma$ (i.e., a collapsing, rotational, or expanding configuration) can be represented by a system $w \in \mathbb{C}^{n}$ satisfying $2 n-1$ real equations corresponding to $n-1$ complex equations

$$
w_{1} V_{k}(w)=w_{k} V_{1}(w) \quad(2 \leqslant k \leqslant n-2) \quad M(w)=0 \quad w_{s}=1
$$

for some s, accompanied by one real equation

$$
S(w)=0
$$

If we additionally fix the value of the real or imaginary part of $w_{r}$ for some $r \neq s$, we obtain a system of $2 n$ real equations. In other words, elements of $\Sigma$ are zeroes of a map $f: \mathbb{C}^{n} \cong \mathbb{R}^{2 n} \rightarrow \mathbb{R}^{2 n}$, whose components, with some $1 \leqslant r \neq s \leqslant n, a \in \mathbb{R}$, can be specified as

$$
\begin{aligned}
& f^{1}(w)=S(w) \\
& f^{2}(w) \text { is either } \operatorname{Re}\left(w_{r}\right)-a \quad \text { or } \operatorname{Im}\left(w_{r}\right)-a \\
& f^{3}(w)+\mathrm{i} f^{4}(w)=w_{s}-1 \\
& f^{5}(w)+\mathrm{i} f^{6}(w)=M(w) \\
& f^{2 k-1}(w)+\mathrm{i} f^{2 k}(w)=w_{1} V_{k}(w)-w_{k} V_{1}(w) \quad \text { for } \quad 4 \leqslant k \leqslant n
\end{aligned}
$$

\section{Approximate solutions of non-linear systems of equations}

Given a system of equations $f(x)=0$, where $f$ is a map from $\mathbb{R}^{n}$ to itself, by a $\rho$-approximate solution we mean a point $y \in \mathbb{R}^{n}$ with the property

$$
\exists x \in \mathbb{R}^{n}: \quad(f(x)=0) \wedge(|x-y|<\rho)
$$

We say that $y \in \mathbb{R}^{n}$ satisfies the equations $\epsilon$-approximately if $|f(y)|<\epsilon$. Numerical methods usually provide points that satisfy equations $\epsilon$-approximately for small $\epsilon$. What we really need are $\rho$-approximate solutions to the equations (with small $\rho$ ). An equation may be satisfied $\epsilon$-approximately at some points for any $\epsilon>0$ but have no solutions whatsoever. This gap can be filled in several ways, one of them being an approach based on the topological degree of a map (cf. Frommer et al., 2007). We shall use the following version of this approach. 
Theorem 2. Let $f: U \rightarrow \mathbb{R}^{n}$ be a $C^{2}$ map defined on an open set $U \subset \mathbb{R}^{n}$. Suppose we have a point $P \in U$ and a matrix $\mathbf{A}$ of degree $n$ which for some real numbers $E, \eta, \rho$ satisfy the following conditions.

- $\left|\sum_{j} A_{j}^{i} \frac{\partial f^{j}}{\partial x^{k} \partial x^{l}}(x)\right| \leqslant E, x \in U, 1 \leqslant i, k, l \leqslant n$,

- $\forall i, k\left|\sum_{j} A_{j}^{i} \frac{\partial f^{j}}{\partial x^{k}}(P)-\delta_{k}^{i}\right| \leqslant \eta<\frac{1}{n}$,

- $0<\rho<\frac{1-n \eta}{n^{2} E}$ and the box $B=B(P, \rho)$ of radius $\rho$ around $P$ is contained in $U$,

- $\forall i \quad\left|\sum_{j} A_{j}^{i} f^{j}(P)\right|<\rho\left(1-n \eta-\rho n^{2} E\right)$.

Then the box $B$ contains a solution of the equation $f(x)=0$.

Observe that if $x$ is a solution of $f(x)=0$ and $\mathbf{D f}$ is invertible at $x$ then the conditions are satisfied with $\eta, \rho$ arbitrarily small if $P$ is close enough to $x$ and $\mathbf{A}$ is a good approximation of $\operatorname{Df}(P)^{-1}$. In our applications, the components of $f$ are rational functions with integer coefficients and $P$ has rational coordinates. It follows that $f(P), \mathbf{D f}(P)$ and $\mathbf{D f}(P)^{-1}$ have rational entries, although the denominators may be huge. In principle, one can take $\mathbf{A}=\mathbf{D f}(P)^{-1}$, but it is more reasonable to take some rational approximation instead. All conditions except the first one are required to hold at $P$ only. Therefore, they can be verified by direct calculation, either by exact calculation of $f$ and its derivatives as rational numbers or by some form of interval arithmetic. Now the first inequality, in opposition to the remaining conditions, should hold at any point of $U$, and therefore needs special treatment.

Proof. The boundary $S=\partial B$ of $B$ is the union of $2 n$ facets

$$
S_{i}^{ \pm}=\left\{y \in \mathbb{R}^{n}: \forall_{k}\left|y^{k}-P^{k}\right| \leqslant \rho, y^{i}=P^{i} \pm \rho\right\}
$$

for $1 \leqslant i \leqslant n$. Consider the map

$$
U \ni x \rightarrow h(x)=\mathbf{A} \circ f(x) \quad h^{i}(x)=\sum_{j} A_{j}^{i} f^{j}(x)
$$

In order to apply the topological degree approach we need to know that $h^{i}$ is positive on $S_{i}^{+}$ and negative on $S_{i}^{-}$. Having assumed that for a moment, we consider the homotopy

$$
H(x, t)=t h(x)+(1-t)(x-P) \quad x \in B \quad t \in[0,1]
$$

between $h$ and translation $x \rightarrow x-P$. For $y \in S_{i}^{ \pm}, y^{i}-P^{i}= \pm \rho$ has the same sign as $h^{i}(y)$. Therefore $H^{i}(y, t)$ is nonzero on $S_{i}^{ \pm}$for all $t$. It follows that $H(y, t) \neq 0$ for $y \in \partial B, t \in[0,1]$, and on $B h$ as the same topological degree as the translation, which is 1 . Nonzero topological degree of $h$ on $B$ implies that $B$ contains a solution of the equation.

It remains to show that $h^{i}$ is indeed nonzero on $S_{i}^{ \pm}$. To this end, we check the following inequalities

$$
\left|\partial_{k} h^{i}(P)-\delta_{k}^{i}\right|=\left|\sum_{j} A_{j}^{i} \partial_{k} f^{j}(P)-\delta_{k}^{i}\right| \leqslant \eta
$$

For any $z \in B$

$$
\begin{aligned}
& \left|\partial_{k} h^{i}(z)-\delta_{k}^{i}\right| \leqslant\left|\partial_{k} h^{i}(z)-\partial_{k} h^{i}(P)\right|+\left|\partial_{k} h^{i}(P)-\delta_{k}^{i}\right| \\
& \quad \leqslant n \rho \sup _{B}\left|\partial_{k l} h^{i}\right|+\eta=n \rho \sup _{B}\left|\sum_{j} A_{j}^{i} \partial_{k l} h^{j}\right|+\eta \leqslant \rho n E+\eta
\end{aligned}
$$


Denote the center of $S_{i}^{ \pm}$by $C=C_{i}^{ \pm}$. Its coordinates are $C^{k}=P^{k} \pm \rho \delta_{k}^{i}$. For $y \in S_{i}^{ \pm}$we have

$$
\begin{aligned}
& \left|h^{i}(y)- \pm \rho\right| \leqslant\left|h^{i}(y)-h^{i}(C)\right|+\left|h^{i}(C)- \pm \rho-h^{i}(P)\right|+\left|h^{i}(P)\right| \\
& \quad \leqslant \rho(n-1) \sup _{B, k \neq i}\left|\partial_{k} h^{i}\right|+\rho \sup _{B}\left|\partial_{i} h^{i}-1\right|+\left|h^{i}(P)\right| \leqslant \rho n(\rho n E+\eta)+\left|h^{i}(P)\right|
\end{aligned}
$$

This is less than $\rho$ since we assume that $\left|h^{i}(P)\right|=\left|\sum_{j} A_{j}^{i} f^{j}(P)\right|<\rho\left(1-n \eta-\rho n^{2} E\right)$. The inequality $\left|h^{i}(y)- \pm \rho\right|<\rho$ implies that $h^{i}(y)$ is positive for $y \in S_{i}^{+}$and negative for $y \in S_{i}^{-}$, as required.

\section{Approximate solutions of O'Neil's equations}

The aim of this Section is to give a criterion which enables one to conclude rigorously that a point satisfying O'Neil's equations approximately is in fact an approximate solution to the equations. Thus the point is close to a true solution to the equations.

Since we plan to apply Theorem 2, we have to bound the second-order derivatives of the components $f^{i}$ of our basic equation system (see (2.5)) with respect to the real independent variables. To deal with them, we prove the following.

Theorem 3. Let $U \subset \mathbb{C}^{n}$ be an open set and

$$
C=\sup _{p \neq q, z \in U}\left|z_{p}\right|+\left|z_{q}\right|<\infty \quad D=\inf _{p \neq q, z \in U}\left|z_{p}-z_{q}\right|>0
$$

Then, for any indices $k, l$, the second-order partial derivatives of the complex-valued function

$$
U \ni z \rightarrow V_{k}(z) z_{l}-V_{l}(z) z_{k} \in \mathbb{C}
$$

with respect to $2 n$ real variables $\operatorname{Re}\left(z_{i}\right), \operatorname{Im}\left(z_{i}\right)$, are bounded by

$$
\sum_{s}\left|\kappa_{s}\right| \frac{12 D+14 C}{D^{3}}
$$

Proof. By definition

$$
V_{k}=\mathrm{i} \sum_{s \neq k} \kappa_{s} \frac{z_{k}-z_{s}}{\left|z_{k}-z_{s}\right|^{2}}
$$

The bounds proved in Lemma 5 give

$$
\left|\partial_{a b} V_{l} z_{k}\right|=\left|\sum_{s \neq l} \kappa_{s} \partial_{a b} \frac{z_{k}\left(z_{l}-z_{s}\right)}{\left|z_{l}-z_{s}\right|^{2}}\right| \leqslant \sum_{s \neq l}\left|\kappa_{s}\right| \frac{6\left|z_{l}-z_{s}\right|+14\left|z_{k}\right|}{\left|z_{l}-z_{s}\right|^{3}} \leqslant \sum_{s}\left|\kappa_{s}\right| \frac{6 D+14\left|z_{k}\right|}{D^{3}}
$$

Therefore

$$
\left|\partial_{a} \partial_{b}\left(V_{k} z_{l}-V_{l} z_{k}\right)\right| \leqslant \sum_{s}\left|\kappa_{s}\right| \frac{12 D+14\left(\left|z_{k}\right|+\left|z_{l}\right|\right)}{D^{3}} \leqslant \sum_{s}\left|\kappa_{s}\right| \frac{12 D+14 C}{D^{3}}
$$


Lemma 5. For indices $k, l$, and $s \neq l$, consider complex-valued functions

$$
\begin{aligned}
& L=z_{k}\left(z_{l}-z_{s}\right)=\left(x_{k} x_{l}-x_{k} x_{s}-y_{k} y_{l}+y_{k} y_{s}\right)+\mathrm{i}\left(x_{k} y_{l}-x_{k} y_{s}+y_{k} x_{l}-y_{k} x_{s}\right) \\
& M=\left|z_{l}-z_{s}\right| \quad M^{2}=\left(x_{l}-x_{s}\right)^{2}+\left(y_{l}-y_{s}\right)^{2} \quad T=z_{k} \frac{z_{l}-z_{s}}{\left|z_{l}-z_{s}\right|^{2}}
\end{aligned}
$$

where $z_{j}=x_{j}+\mathrm{i} y_{j}, x_{j}, y_{j} \in \mathbb{R}$ (for any $j$ ) is understood. We have the following bounds for the derivatives, where $u, w$ denote any of the real variables $x_{j}, y_{j}$

$$
\begin{array}{lll}
|L| \leqslant\left|z_{k}\right| M & \left|\partial_{u} L\right| \leqslant M+\left|z_{k}\right| & \left|\partial_{u} M\right| \leqslant 1 \\
\left|\partial_{u w} L\right| \leqslant 2 & \left|\partial_{u} M \partial_{w} M+M \partial_{u w} M\right| \leqslant 1 & \left|M \partial_{u w} M\right| \leqslant 2
\end{array}
$$

and

$$
\left|\partial_{u w} T\right| \leqslant \frac{6\left|z_{l}-z_{s}\right|+14\left|z_{k}\right|}{\left|z_{l}-z_{s}\right|^{3}}
$$

Proof. We have

$$
\left|\partial_{u} z_{k}\right| \leqslant 1 \quad\left|\partial_{u} z_{l}-\partial_{u} z_{s}\right| \leqslant 1
$$

since $s \neq l$ implies that at most one of the derivatives $\partial_{u} z_{l}, \partial_{u} z_{s}$ can be non-zero. As

$$
\partial_{u} L=\partial_{u}\left(z_{k}\left(z_{l}-z_{s}\right)\right)=\partial_{u} z_{k}\left(z_{l}-z_{s}\right)+z_{k}\left(\partial_{u} z_{l}-\partial_{u} z_{s}\right)
$$

we have

$$
\left|\partial_{u} L\right| \leqslant 1 \cdot\left|z_{l}-z_{s}\right|+\left|z_{k}\right| \cdot 1=M+\left|z_{k}\right|
$$

Similarly $\left|\partial_{u w} L\right| \leqslant 2$. Other relations (4.1) follow from

$$
\begin{aligned}
& \left|2 M \partial_{u} M\right|=\left|\partial_{u}\left(M^{2}\right)\right| \leqslant 2\left|z_{l}-z_{s}\right|=2 M \\
& \left|2 \partial_{w} M \partial_{u} M+2 M \partial_{u w} M\right|=\left|\partial_{u w}\left(M^{2}\right)\right| \leqslant 2
\end{aligned}
$$

Regarding (4.2), the quotient rule gives

$$
\begin{aligned}
& \partial_{u}\left(\frac{L}{M^{2}}\right)=\frac{\partial_{u} L M-2 L \partial_{u} M}{M^{3}} \\
& \partial_{u w}\left(\frac{L}{M^{2}}\right)=\frac{\partial_{w}\left(\partial_{u} L M-2 L \partial_{u} M\right) M-3\left(\partial_{u} L M-2 L \partial_{u} M\right) \partial_{w} M}{M^{4}} \\
& \quad=\frac{\partial_{u w} L M^{2}-2 \partial_{u} L M \partial_{w} M-2 \partial_{w} L M \partial_{u} M-2 L M \partial_{u w} M+6 L \partial_{u} M \partial_{w} M}{M^{4}}
\end{aligned}
$$

Thus (4.2) follows, as the second derivatives of $T$ are bounded by

$$
M^{-4}\left(2 M^{2}+4 M\left(M+\left|z_{k}\right|\right)+4 M\left|z_{k}\right|+6 M\left|z_{k}\right|\right)=M^{-4}\left(6 M^{2}+14 M\left|z_{k}\right|\right)
$$

Now our criterion for the existence of collapsing configurations is a direct application of Theorem 2 with the bound obtained in Theorem 3.

Theorem 4. Consider circulations $\kappa=\left(\kappa_{s}\right)_{s} \in \mathbb{R}^{*}, 1 \leqslant s \leqslant n$. Suppose $P \in \mathbb{C}^{n}$ is a point and $B=B(P, \rho)$ is a box of radius $\rho>0$ centered at $P$. Suppose further that positive real numbers $C, D, E, \eta$, a vector $\mathbf{F} \in \mathbb{R}^{2 n}$ and a matrix $\mathbf{A}$ of degree $2 n$, satisfy (for $f^{i}$ given in (2.5)) the following conditions.

(1) $C \geqslant\left|P^{r}\right|+\left|P^{s}\right|+2 \sqrt{2 n} \rho$ for $1 \leqslant r<s \leqslant n$ 
(2) $D \leqslant\left|P^{r}-P^{s}\right|-2 \sqrt{2 n} \rho$ for $1 \leqslant r<s \leqslant n$

(3) $F_{1} \geqslant 2 \max _{s \leqslant n}\left|\kappa_{s}\right|, F_{j}=0(j=2, \ldots, 6), F_{j}>\frac{12 D+14 C}{D^{3}} \sum_{s \leqslant n}\left|\kappa_{s}\right|(j=7, \ldots, 2 n)$

(4) $\forall i \quad \sum_{j=1}^{2 n}\left|A_{j}^{i}\right| F_{j} \leqslant E$

(5) $\forall i, k \quad\left|\sum_{j=1}^{2 n} A_{j}^{i} \frac{\partial f^{j}}{\partial x^{k}}(P)-\delta_{k}^{i}\right| \leqslant \eta<\frac{1}{2 n}$

(6) $\rho<\frac{1-2 n \eta}{4 n^{2} E}$

(7) $\forall i \quad\left|\sum_{j=1}^{2 n} A_{j}^{i} f^{j}(P)\right|<\rho\left(1-2 n \eta-4 \rho n^{2} E\right)$.

Then the box $B$ contains a solution to O'Neil's equations for the circulation $\kappa$.

Proof. It is crucial to see that the first three conditions guarantee that for $x \in B$ and any indices $j, k, l \leqslant 2 n$

$$
\left|\frac{\partial f^{j}}{\partial x^{k} \partial x^{l}}(x)\right| \leqslant F_{j}
$$

Then the remaining four conditions correspond to the four conditions in Theorem 2 and the conclusion follows.

To check the bounds, observe that five of the components of $f$ are linear, so that their secondorder derivatives vanish. Those five components are: the real and imaginary part of $M(w)$, the real and imaginary part of $w_{s}-1$ (for some $s$ ), and $\operatorname{Re}\left(w_{r}\right)-a$ or $\operatorname{Im}\left(w_{r}\right)-a$ (for some $r$ ). According to our numbering, they correspond to $F_{j}$ for $j=2, \ldots, 6$. One (real) component, namely

$$
S(w)=\sum_{i} \kappa_{i}\left|w_{i}\right|^{2}
$$

is quadratic, and the bound is $F_{1}=2 \max \left|\kappa_{s}\right|$. The other components are the real and imaginary part of expressions of the form $w_{k} V_{l}(w)-w_{l} V_{k}(w)$. We use bounds established in Theorem 3 , with $C$ and $D$ defined there. A direct expression for $C$ and $D$, given in the first two conditions above, is obvious: as $z \in U=B(P, \rho)$, we have

$$
\begin{aligned}
& \left|z_{r}\right|+\left|z_{s}\right| \leqslant\left|z_{r}-P^{r}\right|+\left|P^{r}\right|+\left|z_{s}-P^{s}\right|+\left|P^{s}\right| \leqslant\left|P^{r}\right|+\left|P^{s}\right|+2 \sqrt{2 n \rho^{2}} \\
& \left|z_{r}-z_{s}\right| \geqslant\left|P^{r}-P^{s}\right|-\left|P^{r}-z_{r}\right|-\left|P^{s}-z_{s}\right| \geqslant\left|P^{r}-P^{s}\right|-2 \sqrt{2 n \rho^{2}}
\end{aligned}
$$

\section{The applicability limits of the criterion}

Suppose that a vortex system $P$ satisfies the discrete O'Neil's equations up to certain accuracy. If the accuracy is low, we expect that the criterion is not able to detect a collapsing system in any neighbourhood of $P$. If accuracy is higher, the criterion should respond positively and supply an upper bound for the distance from $P$ to the nearest collapsing configuration $Q$. When the accuracy becomes even higher (and presumably the actual distance from $P$ to $Q$ goes to zero), the bound supplied by the criterion should also tend to zero.

In order to illustrate how our criterion works in such a situation, consider a simple system of four vortices. We shall use the well-known analytical solution of Novikov. His results imply in particular that for $\lambda=2+\sqrt{3}$ all systems of the form

$$
(-w,-1, w, 1)
$$


satisfying $\lambda|w|^{2}=1$ are collapsing systems for the circulation

$$
\kappa=(\lambda,-1, \lambda,-1)
$$

Let us choose a sample collapsing system

$$
Q=(-\mu(1-\mathrm{i}),-1, \mu(1-\mathrm{i}), 1)
$$

with $\mu=\frac{\sqrt{2-\sqrt{3}}}{\sqrt{2}}$ and a one-parameter family of systems

$$
Z^{t}=(-\mu(1-\mathrm{i}),-1, \mu(1-\mathrm{i})+t, 1)
$$

which for $t=0$ gives the collapsing system. The distance from $Z^{t}$ to $Q$ in the $L^{\infty}$ metric is $t$. Our aim is to see whether our criterion is able to detect a collapsing system at some distance from $Z^{t}$.

In the notation of (2.5) we take $f^{2}(w)=\operatorname{Im}\left(w_{1}\right)-\mu$ (thus $r=1, a=\mu$ ) and $f^{3}(w)+\mathrm{i} f^{4}(w)=w_{4}-1$ (so that $s=4$ ). Clearly, along $Z^{t}$ the functions $f^{i}$ vanish for $i=2, \ldots, 4,6$, while $f^{1}=S=\lambda t(t+2 \mu)$ and $f^{5}=\operatorname{Re}(M)=\lambda t$, with some more complicated formulas for $f^{7}$ and $f^{8}$. They are rational functions in $t$ with simple zero at 0 , as the equations $f^{i}=0$ are satisfied at $Z^{0}$. The points $Z^{t}$ for $t \neq 0$ fail to satisfy the equations with the error illustrated in Fig. 1, where the components of $f^{i}\left(Z^{t}\right)$, divided by $t$, are pictured.

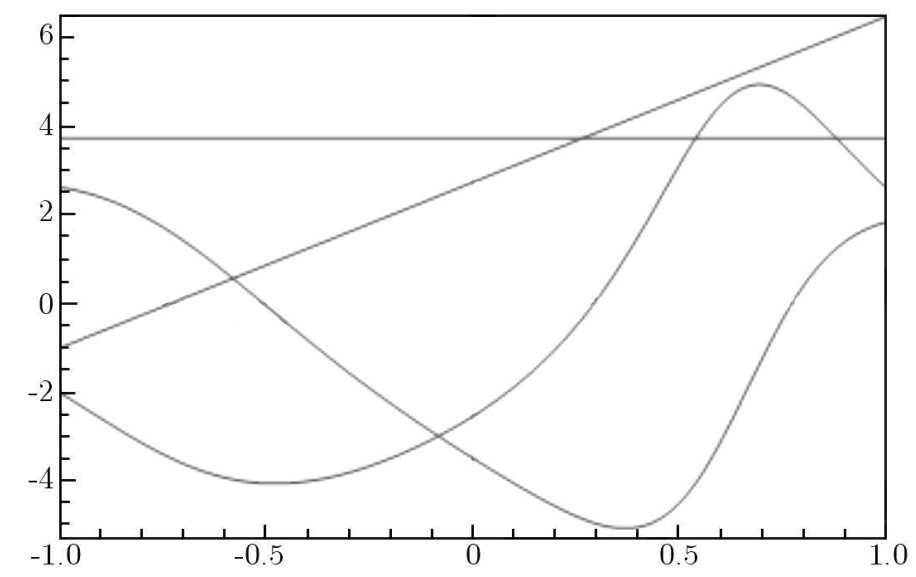

Fig. 1. Coordinates of $f\left(Z^{t}\right) / t$

In order to investigate the possibility of applying the criterion for the values of the parameter $t$ distant from zero, let us estimate the constants appearing in the criterion in an approximate way, that is for $t \approx 0, \rho \approx 0$. On this assumption, the values of $C, D, \mathbf{F}$ can be simply evaluated at the point $Q$. Therefore

$$
C=2.0 \quad D=0.535898 \quad F_{1}=7.4641 \quad F_{7}=F_{8}=2968.59
$$

and the remaining coordinates of $\mathbf{F}$ are zero. Furthermore, we assume that $\mathbf{A}$ is a good approximation of the matrix $\mathbf{D f}^{-1}$ calculated at $Q$ too. Thus

$$
\eta=0 \quad E=5544.77
$$

Now Condition 6 in $(2.5)$ says that

$$
\rho<2.9415 \cdot 10^{-6}
$$


Indeed, the criterion works only when the tested point is close to the collapsing point. Moreover, Condition 7 , with $\eta=0$, says that $\left|\sum_{j} A_{j}^{i} f^{j}\right|$ must be less than $\rho$ for each $i$. It turns out that $\max _{i}\left|\sum_{j} A_{j}^{i} f^{j}(t)\right| \approx|t|$. Thus the criterion cannot be applied for $|t|>2.81797 \cdot 10^{-6}$.

Now we want to see that the criterion does work for small values of $t$. The values of $C, D$, $\mathbf{F}, E, \mathbf{A}$, occurring in our criterion, change only slightly if the above approximate calculations (at $Q=Z^{0}$ ) are replaced by exact estimations taking into account the fact that the calculated values depend on $t$ as well as on $\rho$. If we confine ourselves to the values of $t, \rho$ suggested by the above estimate (for example we assume that $|t|<10^{-6}, \rho<10^{-6}$ ), then the estimation $C=2.0, D=0.535898$ used above, applicable only at the limit point $Q$, may be replaced by one which is only slightly weaker, but now valid along the full range of $t \in\left[-10^{-6}, 10^{-6}\right]$. As the impact of these changes on further calculations is negligible, we conclude that the resulting inequalities hold with certainty throughout this interval.

In the sequel, we restrict ourselves to the study of points $P=Z^{t}$ for $t \in\left[-10^{-6}, 10^{-6}\right]$. Regarding Conditions 1 and 2, we can assume

$$
C=2.0+5.6569 \rho \quad D=0.5358-5.6569 \rho
$$

Thus the criterion does not work if $0.5358-5.6569 \rho<0$, that is for $\rho>0.09472$. We see again that the criterion does not work for large $\rho$, therefore we will certainly not be able to use it to determine the existence of the collapsing systems unless the tested point $P$ is sufficiently close to such an arrangement. We assume $\rho<10^{-6}$, therefore we can take $C=2.0001, D=0.5357$. According to Condition 3 , we can take $F_{1}=7.4642, F_{7}=F_{8}=2119.59$, and the remaining $F_{j}$ (i.e., for $j=2, \ldots, 6)$ can be taken as zero. As before, $\mathbf{A}=\mathbf{A}(t)$ can be a good approximation of the inverse of the matrix $\mathbf{D f}$ at the point $Z^{t}$, so that practically $\eta \approx 0$. Now Condition 4 gives a bound for $E$. The function $\max _{i}\left(\sum_{j}\left|A_{j}^{i}\right| F^{j}\right)$ can be well approximated by $3962.15+15840.8 t$, so that one can take $E=3962.16$. Condition 6 needs $\rho<1 / 4 n^{2} E \approx 3.94356 \cdot 10^{-6}$, which is satisfied. Now in Condition 7 the factor $1-2 n \eta-4 \rho n^{2} E$ is about 0.746422 . Thus the condition will be satisfied if for any $i$

$$
\left|\sum_{j} A_{j}^{i} f^{j}(P)\right|<0.7464 \rho
$$

The individual sums $\sum_{j} A_{j}^{i} f^{j}(P)$ at the variable point $P=Z^{t}$ for $t \in[-0.01,01]$ behave as shown in Fig. 2. Note that it does not differ much from Fig. 1, where a constant matrix $\mathbf{A}(0)$ is

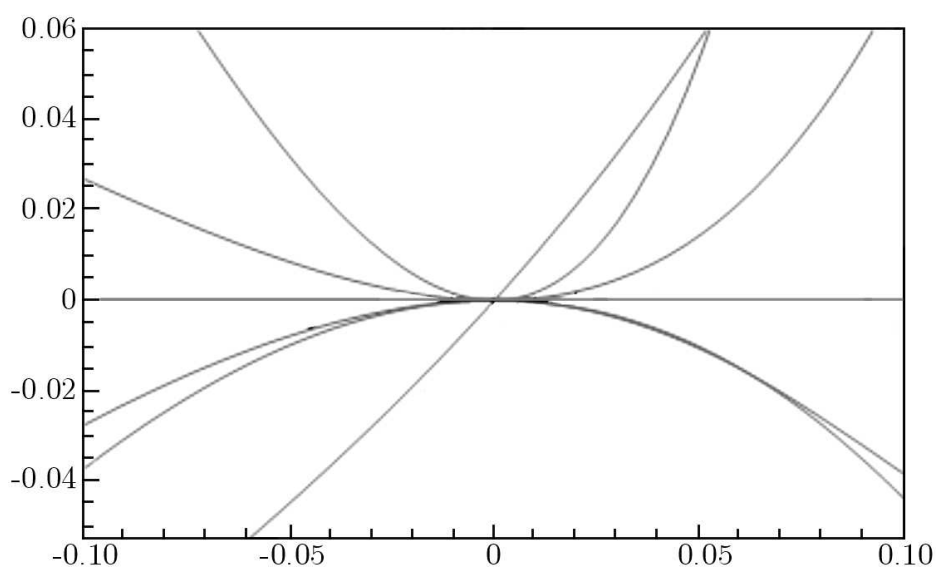

Fig. 2. Coordinates of $A(t) f(t),|t|<0.1$

used. The maximum absolute value of these expressions in this interval is less than $1.03|t|$, and for $|t|<10^{-6}$ it is even less than $1.000003|t|$. Thus the criterion implies that for such $t$, the 
distance from the points $Z^{t}$ to the collapsing configurations set is smaller than $1.3398|t|$. Let us recall that in fact the collapsing point $Q=Z^{0}$ is precisely at the distance $|t|$ from $Z^{t}$. One can conclude that the criterion very well fulfills the task of detecting collapsing systems located close to the tested point.

\section{A collapsing system of seven vortices}

The very reason why the paper has been written is to provide a way to argue rigorously that a numerically found system which allegedly approximates a collapsing system really does so. We shall show now how our criterion works for the seven-vortex system discussed in Lewkowicz and Kudela (2012). The circulation is

$$
\kappa=(2,2,-4,-4,-4,-4,3)
$$

We have $L=0$ and $\sigma=-9 \neq 0$, so that the necessary condition on $\kappa$ for the existence of collapsing configurations is fulfilled. We add the equation $z_{7}=1$ to our equation system, which means that $s=7$ in notation of Formula (2.5). Recall that a point where O'Neil's equations are satisfied approximately has been obtained in the following way. By a random choice of the initial point and applying the steepest descent method we find that the point

$$
\begin{aligned}
P= & (-1.31+10.00 \mathrm{i}, 4.51+5.39 \mathrm{i},-1.27+7.59 \mathrm{i}, 1.21+2.06 \mathrm{i}, \\
& -0.58-1.00 \mathrm{i}, 2.99-0.95 \mathrm{i}, 1.00+0.00 \mathrm{i})
\end{aligned}
$$

has $|f(P)|^{2}=0.652917$, while random points in the neighborhood $B(P, 0.01)$ may have $|f|^{2}$ as big as $3.88 \cdot 10^{6}$. Therefore, we hope that this point may be close enough to the solution set. We choose to fix one of the real coordinates and complete our set of equations with $y_{1}=\operatorname{Im}\left(z_{1}\right)=10$ $(r=1$ in (2.5)). The system $f(z)=0$ (with $f$ as in (2.5)) has fourteen equations now

$$
\begin{aligned}
\mathbb{R}^{14} \approx \mathbb{C}^{7} \ni z \rightarrow f(z)=\left(\sum_{k} \kappa_{k}\left|z_{k}\right|^{2}, \operatorname{Im}\left(z_{1}\right)-10, z_{7}-1, \sum_{k} \kappa_{k} z_{k},\right. \\
\left.V_{1} z_{4}-V_{4} z_{1}, V_{1} z_{5}-V_{5} z_{1}, V_{1} z_{6}-V_{6} z_{1}, V_{1} z_{7}-V_{7} z_{1}\right) \in \mathbb{R}^{2} \times \mathbb{C}^{6} \approx \mathbb{R}^{14}
\end{aligned}
$$

We can continue with the steepest descent method and arrive at the point $Q$, where the value $|f(Q)|^{2}=1.65 \cdot 10^{-6}$. The values $y_{1}$ and $z_{7}$ are kept fixed and the other coordinates change by less than 0.01. A measure of quality of the obtained point is the value $\omega=\left(V_{k}-V_{l}\right) /\left(z_{k}-z_{l}\right)$, which should be independent of $k, l$ and have a negative real part for the system to collapse. At $Q$, the real part of $\omega$ varies between -0.02625 and -0.02595 , and $\operatorname{Im}(\omega)$ is in $[-0.23185,-0.23118]$.

Now we change the method of seeking a solution to one of Newton's equations, which allows us to obtain a point where the equations are satisfied with the accuracy practically as high as the precision used in the calculations. With the standard machine precision of around 18 digits we can get immediately a point $R$ with $|f(R)|^{2} \leqslant 10^{-34}$ and with 17 accurate digits in $\operatorname{Re}(\omega)$. The expected collapse time is $t_{\infty}=\frac{-1}{2 \operatorname{Re} \omega} \approx 19.1806$. In case higher accuracy is needed, we can switch from the standard machine precision to the high precision provided by software. With suitable precision, Newton's method immediately can produce the point $R$ with the first coordinate equal to

$$
x_{1} \approx-1.3127662694757482864411913449969096988121871876601
$$

In order to prove that this is an approximate solution to O'Neil's equations, we shall apply the criterion from Theorem 4 . We have to specify all constants appearing in it as well as the matrix A. We have 


$$
\max _{p \neq q}\left|R^{p}\right|+\left|R^{q}\right| \approx 17.791133 \quad \min _{p \neq q}\left|R^{p}-R^{q}\right| \approx 1.880368
$$

We shall be interested only in $\rho<0.01$. Therefore, we can take $C=17.87, D=1.80$ in order to satisfy Conditions 1 and 2 in Theorem 4 . Since $\max \left|\kappa_{s}\right|=4$ and $\sum_{s}\left|\kappa_{s}\right| \frac{12 D+14 C}{D^{3}} \approx 1071.83$, for (3) we can take

$$
F_{1}=8.00 \quad F_{k}=\left\{\begin{array}{lll}
0 & \text { for } & k=2, \ldots, 6 \\
1072.0 & \text { for } & k=7, \ldots, 14
\end{array}\right.
$$

As the matrix $\mathbf{A}$ we take the inverse of $\mathbf{D f}(R)$ rounded to 5 decimal digits. Thus the entries of $\mathbf{A}$ are rational decimals. With this $\mathbf{A}$, we calculate that the values of $E$ and $\eta$ suitable for (4) and (5) are

$$
E=30995.5 \quad \eta=0.00029
$$

Now the bound for $\rho$ in $(6)$ is

$$
\rho<\frac{1-2 n \eta}{4 n^{2} E}=4.74 \cdot 10^{-6}
$$

For $\rho=10^{-6}$ we have

$$
\rho\left(1-n \eta-\rho n^{2} E\right) \approx 7.85828 \cdot 10^{-7}
$$

This means that final Condition 7 is

$$
\left|\sum_{j} A_{j}^{i} f^{j}(P)\right| \leqslant 7.85828 \cdot 10^{-7}
$$

for any $i$. In fact, the actual maximal value of $\left|\sum_{j} A_{j}^{i} f^{j}(P)\right|$ for our high accuracy solution is $\epsilon \approx 1.69036 \cdot 10^{-49}$. This means that the conditions are satisfied and our criterion guarantees that a true collapsing system exists within a distance smaller than $\rho=10^{-6}$ from $R$. Moreover, for $\rho<10^{-12}$, with $\eta=0.00028$, we have $1-2 n \eta-4 \rho n^{2} E>0.995$ and $\rho$ can be practically taken as small as $\epsilon$. We conclude that in fact the error of our approximate solution is less than $1.70 \cdot 10^{-49}$.

\section{Acknowledgments}

I am deeply grateful to the Referee for suggesting that an example illustrating how the accuracy of the approximate solution influences the applicability of the criterion should be included in the paper. Section 5 is an attempt to fulfill this suggestion.

\section{References}

1. Aref H., Rott N., Thomann H., 1992, Groebli's solution of the three-vortex problem, Annual Review of Fluid Mechanics, 24, 1-21

2. Frommer A., Hoxha F., Lang B., 2007, Proving the existence of zeros using the topological degree and interval arithmetic, Journal of Computational and Applied Mathematics, 199, 397-402

3. Garduno A., Lacomba E.A., 2007, Collisions and regularization for the 3-vortex problem, Journal of Mathematical Fluid Mechanics, 9, 75-86

4. Groebli W., 1877, Spezielle Probleme uber die Bewegung geradliniger paralleler Wirbelfaden, Vierteljahrsschr. Naturforsch. Ges. Zürich, 22, 129 
5. Helmholtz H., 1858, Uber die Integrale der hydrodynamischen Gleichungen, welche Wirbelbewegungen entsprechen, Journal für die reine und angewandte Mathematik, 55, 25-55

6. Lewkowicz M., Kudela H., 2012, Collapse of $N$ vortices, [In:] XX Fluid Mechanics Conference, Gliwice, Poland, 17-20 September, Silesian University of Technology. Institute of Power Engineering and Turbomachinery

7. Novikov E.A., Sedov Yu.B., 1979, Vortex collapse, Soviet Physics - Journal of Experimental and Theoretical Physics, 50, 297

8. O'NeIL K.A., 1987, Stationary configurations of point vortices, Transactions of the American Mathematical Society, 302, 2, 383-425

Manuscript received March 11, 2013; accepted for print June 3, 2014 